\title{
Foliations of Hyperbolic Space by Constant Mean Curvature Surfaces Sharing Ideal Boundary
}

\author{
David Chopp and John A. Velling
}

\section{CONTENTS}

1. Introduction and Background

2. Mean Curvature of Level Sets

3. The PDE and the Flow

4. Limitations

5. The Numerical Method

6. Examples

7. Conclusion
Let $\gamma$ be a Jordan curve in $\mathbf{S}^{2}$, considered as the ideal boundary of $\mathbf{H}^{3}$. Under certain circumstances, it is known that for any $c \in(-1,1)$, there is a disc of constant mean curvature $c$ embedded in $\mathbf{H}^{3}$ with $\gamma$ as its ideal boundary. Using analysis and numerical experiments, we examine whether or not these surfaces in fact foliate $\mathbf{H}^{3}$, and to what extent the known conditions on the curve can be relaxed.

\section{INTRODUCTION AND BACKGROUND}

In the paper [Guan and Spruck 00], Guan and Spruck consider surfaces of constant mean curvature $(\mathrm{cmc})$ in $\mathbf{H}^{3}$. They show that certain curves in the ideal boundary $\mathbf{S}_{\infty}^{2}$ of $\mathbf{H}^{3}$ are spanned by embedded cmc discs for a range of mean curvature values. This article examines whether or not the surfaces obtained by Guan-Spruck actually constitute a foliation of $\mathbf{H}^{3}$. This is done by somewhat different methods than those employed in [Guan and Spruck 00], and while an analytical proof is still incomplete, the evidence is that

1. the surfaces of [Guan and Spruck 00] foliate $\mathbf{H}^{3}$,

2. a significantly wider class of curves admit such a foliation, and

3. these foliations can be obtained as the steady-state solution of a nonlinear degenerate parabolic flow.

To be more precise, let $\gamma$ denote a Jordan curve in the complex plane, C. We think of the extended plane, $\hat{\mathbf{C}}$, as the ideal boundary sphere $\mathbf{S}_{\infty}^{2}$ of $\mathbf{H}^{3}$ with a designated point $(\infty)$. Guan and Spruck [Guan and Spruck $00]$ consider the case where $\gamma$ is smooth and star-shaped. In particular, letting $0 \in \mathbf{C}$ be the center of the interior star-shaped region determined by $\gamma$, they demand that $\gamma$ be a radial graph and that the tangent to $\gamma$ never be radial. They show that for any $H \in(-1,1)$, there is a cmc disc of mean curvature $H$ spanning $\gamma$.

(c) A K Peters, Ltd. $1058-6458 / 2003 \$ 0.50$ per page Experimental Mathematics 12:3, page 339 
The technique of Guan-Spruck was to look at the constant mean curvature graph equation in special coordinates dictated by the hypothesis of star-shapedness. They coordinatize $\mathbf{H}^{3}$ by $\mathbf{D} \times \mathbf{R}$, where the discs are the rotationally symmetric geodesic planes separating 0 and $\infty$, and the lines are always orthogonal to these planes. For any $H \in(-1,1)$, they show existence and uniqueness of solutions within the family of graphs. (N.B. More recently the existence and uniqueness of a prescribed mean curvature graph has been established in this setting if there is some $\epsilon>0$ such that $|H|<1-\epsilon$ [Nitsche 01].)

In this setting, for distinct values of $H$, an application of the maximum principle tells us that the discs are nonintersecting. One naturally imagines that the family of surfaces arising as $H$ takes on all different values, actually foliates $\mathbf{H}^{3}$. The only difficulty might be gaps between leaves.

We have numerically and graphically examined the level sets for various star-shaped curves. Evidence supports intuition here: We see no gap between surfaces, and it appears that we do indeed have a foliation. We have extended this investigation to more complicated curves and see similar phenomena - foliations by constant mean curvature surfaces sharing an ideal boundary.

If $\gamma$ is taken to be a more general oriented Jordan curve in $\mathbf{S}_{\infty}^{2}$, it is reasonable to ask whether or not it is the ideal boundary of an embedded cmc disc in $\mathbf{H}^{3}$. Let $\mathbf{S}_{\infty}^{2} \backslash \gamma=\Omega_{+} \cup \Omega_{-}$, where $\Omega_{+}$is the interior of $\gamma$ with the given orientation, and $\Omega_{-}$is the exterior. In [Velling 99], the second author shows that one may properly consider $\Omega_{ \pm}$as simply connected discs of constant mean curvature \pm 1 . And for the case where $\gamma$ is a Euclidian circle in $\mathbf{S}_{\infty}^{2}$, one sees that the spherical caps spanning $\gamma$ have mean curvature between -1 and 1 . Thus, we consider it reasonable to ask for $\mathrm{cmc}$ discs in $\mathbf{H}^{3}$ only when $-1<$ $H<1$.

Our method is to consider a degenerate elliptic PDE, the solution to which, at least where the gradient is nonvanishing, has level sets of constant mean curvature. To study solutions to this PDE, we consider a flow arising from the corresponding degenerate parabolic PDE, for which the steady state is a solution to our degenerate elliptic equation.

The key to obtaining a foliation is to produce an appropriate function on $\mathbf{H}^{3}$ whose gradient is never vanishing. Its level sets then, for topological reasons, form a foliating family of discs.

It is worth noting that the curves we have examined are fairly simple, being for the most part quite symmetric. We have tried more complicated curves, but run into limitations imposed by time and computational feasibility. Thus we have opted, in the nonstar-shaped case, for curves approximating annuli or exhibiting some spiraling.

\section{MEAN CURVATURE OF LEVEL SETS}

Here we derive by rather straightforward computation the expression for the mean curvature of the level set of a regular function on a Riemannian manifold furnished with a conformally flat metric. In particular, let $\mathcal{M}$ denote such a manifold of dimension $n+1$, with metric

$$
d s^{2}=\rho^{2}\left(d x_{0}^{2}+d x_{1}^{2}+\cdots+d x_{n}^{2}\right) .
$$

We use $\nabla F$ and $\Delta F$ for the Euclidean gradient and Laplacian of $F$. On $\mathcal{M}$, the connection is given by

$$
\begin{aligned}
\nabla_{\partial_{i}} \partial_{i} & =\rho^{-1}\left(2 \rho_{i} \partial_{i}-\sum_{0}^{n} \rho_{j} \partial_{j}\right) \\
(i \neq j) \quad \nabla_{\partial_{i}} \partial_{j} & =\rho^{-1}\left(\rho_{j} \partial_{i}+\rho_{i} \partial_{j}\right) .
\end{aligned}
$$

Thus, if we have a function $F: \mathcal{M} \rightarrow \mathbf{R}$ which is regular in a neighborhood of a point $p \in \mathcal{M}$ with $F(p)=c$ and $F_{0}(p) \neq 0$, we derive an expression for the trace of the second fundamental form of the level set of $F$ through $p$.

The unit normal $\nu$ to the level set $F=c$ at $p$ is

$$
\nu=\frac{\nabla F}{\rho|\nabla F|},
$$

and the tangent space to the level set is spanned by the $n$ vectors

$$
X_{i}=-F_{i} \partial_{0}+F_{0} \partial_{i} .
$$

The metric on the level set is thus given by the $n \times n$ matrix

$$
\rho^{2}\left(\begin{array}{ccccc}
F_{0}^{2}+F_{1}^{2} & F_{1} F_{2} & F_{1} F_{3} & \cdots & F_{1} F_{n} \\
F_{1} F_{2} & F_{0}^{2}+F_{2}^{2} & F_{2} F_{3} & \cdots & F_{2} F_{n} \\
\vdots & & \ddots & & \vdots \\
\vdots & & & \ddots & \vdots \\
F_{1} F_{n} & \cdots & \cdots & & F_{0}^{2}+F_{n}^{2}
\end{array}\right) .
$$

The determinant of this matrix is $\rho^{2 n} F_{0}^{2(n-1)}|\nabla F|^{2}$, and the inverse of the matrix is

$$
\begin{aligned}
& \frac{1}{\rho^{2} F_{0}^{2}|\nabla F|^{2}} \times \\
& \left(\begin{array}{cccc}
F_{0}^{2}+\sum_{k \neq 0,1} F_{k}^{2} & -F_{1} F_{2} & \ldots & -F_{1} F_{n} \\
-F_{1} F_{2} & F_{0}^{2}+\sum_{k \neq 0,2} F_{k}^{2} & \cdots & -F_{2} F_{n} \\
\vdots & & \ddots & \\
-F_{1} F_{n} & \ldots & & F_{0}^{2}+\sum_{k \neq 0, n} F_{k}^{2}
\end{array}\right) .
\end{aligned}
$$


We also compute the connection on the tangent space to the level set:

$$
\begin{aligned}
\nabla_{X_{i}} X_{i}= & \nabla_{\left(-F_{i} \partial_{0}+F_{0} \partial_{i}\right)}\left(-F_{i} \partial_{0}+F_{0} \partial_{i}\right) \\
= & \left(F_{i} F_{0 i}-F_{0} F_{i i}\right) \partial_{0}+\left(F_{0} F_{0 i}-F_{i} F_{00}\right) \partial_{i} \\
& +\frac{1}{\rho}\left[\left(2 F_{i}^{2} \rho_{0}-F_{0} F_{i} \rho_{i}\right) \partial_{0}\right. \\
& +\left(2 F_{0}^{2} \rho_{i}-F_{0} F_{i} \rho_{0}\right) \partial_{i} \\
& \left.-\left(F_{0}^{2}+F_{i}^{2}\right) \sum_{0}^{n} \rho_{k} \partial_{k}\right]
\end{aligned}
$$

$$
\begin{aligned}
(i \neq j) & \\
\nabla_{X_{i}} X_{j}=( & \left.F_{i} F_{0 j}-F_{0} F_{i j}\right) \partial_{0}+\left(F_{0} F_{0 i}-F_{i} F_{00}\right) \partial_{j} \\
& +\frac{1}{\rho}\left[\left(2 F_{i} F_{j} \rho_{0}-F_{0} F_{i} \rho_{j}-F_{0} F_{j} \rho_{i}\right) \partial_{0}\right. \\
& +\left(F_{0}^{2} \rho_{j}-F_{0} F_{j} \rho_{0}\right) \partial_{i} \\
& \left.+\left(F_{0}^{2} \rho_{i}-F_{0} F_{i} \rho_{0}\right) \partial_{j}-F_{i} F_{j} \sum_{0}^{n} \rho_{k} \partial_{k}\right]
\end{aligned}
$$

From this, we see that the second fundamental form is given as follows:

$$
\begin{aligned}
-\left\langle\nu, \nabla_{X_{i}} X_{i}\right\rangle= & \frac{\rho}{|\nabla F|}\left[F_{0}^{2} F_{i i}-2 F_{0} F_{i} F_{0 i}\right. \\
& \left.+F_{i}^{2} F_{00}+\left(F_{0}^{2}+F_{i}^{2}\right) \sum_{0}^{n} \frac{\rho_{k}}{\rho} F_{k}\right]
\end{aligned}
$$

$$
\begin{aligned}
& (i \neq j) \\
& -\left\langle\nu, \nabla_{X_{i}} X_{j}\right\rangle=\frac{\rho}{|\nabla F|}\left[F_{0}^{2} F_{i j}-F_{0} F_{i} F_{0 j}-F_{0} F_{j} F_{0 i}\right. \\
& \left.+F_{i} F_{j} F_{00}+F_{i} F_{j} \sum_{0}^{n} \frac{\rho_{k}}{\rho} F_{k}\right] .
\end{aligned}
$$

The mean curvature, $H$, is thus determined by $n H$ being the trace of the inverse of the level set metric times the level set connection. This simplifies to

$n H=\frac{1}{\rho|\nabla F|^{3}}\left[|\nabla F|^{2} \Delta F+n|\nabla F|^{2} \frac{\rho_{k}}{\rho} F_{k}-F_{i} F_{j} F_{i j}\right]$.

Special Case: In the case when $\mathcal{M}=\mathbf{H}^{n+1}$ with an upper half-space model, so that its metric is

$$
\frac{d x_{0}^{2}+d x_{1}^{2}+\cdots+d x_{n}^{2}}{x_{0}^{2}}
$$

and so that $\rho=\frac{1}{x_{0}}$, we have

$$
n H=\frac{x_{0}}{|\nabla F|^{3}}\left[|\nabla F|^{2} \Delta F-\frac{n}{x_{0}}|\nabla F|^{2} F_{0}-F_{i} F_{j} F_{i j}\right] .
$$

\section{THE PDE AND THE FLOW}

\subsection{The PDE}

To obtain a foliation of $\mathbf{H}^{3}$ by constant mean curvature surfaces, we will try to obtain a regular function $F$ : $\mathbf{H}^{3} \rightarrow \mathbf{R}$ with level sets yielding the desired surfaces. Thus, using an upper half-space model with coordinates $\left(x_{0}, x_{1}, x_{2}\right)$, we have the metric

$$
\frac{d x_{0}^{2}+d x_{1}^{2}+d x_{2}^{2}}{x_{0}^{2}} .
$$

As determined above, if $F$ is sufficiently smooth and $p \in$ $\mathbf{H}^{3}$ is regular, then the mean curvature of the level set through $p$ is

$$
H=\frac{1}{2} \frac{x_{0}|\nabla F|^{2} \Delta F-x_{0} F_{i} F_{j} F_{i j}-2 F_{0}|\nabla F|^{2}}{|\nabla F|^{3}} .
$$

Thus, if $A: \mathbf{R} \rightarrow \mathbf{R}$ and $F: \mathbf{H}^{3} \rightarrow \mathbf{R}$ satisfying

$$
\frac{1}{2} \frac{x_{0}|\nabla F|^{2} \Delta F-x_{0} F_{i j} F_{i} F_{j}-2 F_{0}|\nabla F|^{2}}{|\nabla F|^{3}}-A(F)=0,
$$

where the expression $A(F)$ is constant where $F$ is constant, then the level sets of $F$ have constant mean curvature. In particular, letting $A$ be the identity function, if $p \in \mathbf{H}^{3}$ is a regular point of $F$ and $F$ satisfies

$$
\frac{1}{2} \frac{x_{0}|\nabla F|^{2} \Delta F-x_{0} F_{i j} F_{i} F_{j}-2 F_{0}|\nabla F|^{2}}{|\nabla F|^{3}}-F=0,
$$

then the mean curvature of the level set of $F$ through $p$ is precisely $F(p)$.

Our goal will be to find a function $F: \mathbf{H}^{3} \rightarrow(-1,1)$ satisfying

1. $F \in C^{2}\left(\mathbf{H}^{3}\right)$,

2. $F(\mathbf{x}) \rightarrow 1(-1)$ as $\mathbf{x} \rightarrow \Omega_{+}\left(\Omega_{-}\right)$radially,

3. $F$ is everywhere regular in $\mathbf{H}^{3}$, and

4. F satisfies the degenerate elliptic PDE (3-1) above.

We call this our Boundary Value Problem (BVP). Conditions 3 and 4 tell us that, at least locally, the level sets of $F$ determine a foliation by cmc surfaces. To deduce that this foliation extends globally, we inject the topological conditions mentioned in Section 1.

\subsection{Topological Considerations}

To put this to work, we need a couple of observations.

Suppose that $F: \mathbf{H}^{3} \rightarrow(-1,1)$ is a solution to the BVP. Then the gradient field is integrable, and the integral curves may all be parameterized to start at $\Omega_{-} \cup \gamma$ 
and end at $\Omega_{+} \cup \gamma$. The following lemmas indicate why the level sets of $F$ are discs and foliate $\mathbf{H}^{3}$. The proofs follow the fourth lemma.

Lemma 3.1. Each value $t \in(-1,1)$ is achieved.

Lemma 3.2. For any $t \in(-1,1), F^{-1}(t)$ has one path component.

Lemma 3.3. If $\left\{\mathbf{x}_{n}\right\}_{n=1}^{\infty}$ is a sequence in $F^{-1}(t)$ for some $t \in(-1,1)$, all of its accumulation points are either in $F^{-1}(t)$ or in $\gamma$. If the sequence leaves compact sets of $F^{-1}(t)$, then all accumulation points are in $\gamma$. All points of gamma can be so accessed.

Lemma 3.4. All of the level sets of $F$ are topological discs.

Proof of Lemmas 3.1-3.4: For any continuous path originating in and orthogonal to $\Omega_{-}$and terminating in and orthogonal to $\Omega_{+}$, the intermediate value theorem gives us that each $t \in(-1,1)$ is assumed by $F$ somewhere along the path.

Now suppose that for some $t \in(-1,1)$ there was more than one component to $F^{-1}(t)$. Take any path in $\mathbf{H}^{3}$ starting on one path component, terminating on another, and transverse to $F$. (By standard position arguments this is always possible.) Then $F$ assumes either a maximum or minimum, distinct from $t$, along this path. At such a point, $D F$ vanishes.

For $t \in(-1,1)$, let $\left\{\mathbf{x}_{n}\right\}_{n=1}^{\infty}$ be a sequence in $F^{-1}(t)$. If this sequence has an accumulation point $\mathbf{x} \in \mathbf{H}^{3}$, then $F(\mathbf{x})=t$. Any other accumulation point must be in $\mathbf{S}_{\infty}^{2}$. Condition 2 of BVP implies that such an accumulation point can be in neither $\Omega_{-}$nor in $\Omega_{+}$. Hence, any accumulation point $\mathbf{x} \notin \mathbf{H}^{3}$ must be in $\gamma$.

To see that all $\mathbf{x} \in \gamma$ are accessed as limits of some sequence in $F^{-1}(t)$, let $\left\{\theta^{+}\right\}_{n=1}^{\infty}$ and $\left\{\theta^{-}\right\}_{n=1}^{\infty}$ be two sequences of points in $\Omega_{+}$and $\Omega_{-}$, respectively, with $\mathbf{x}$ as their limits. Let $\mathcal{C}_{n}$ be the hyperbolic geodesics from $\theta_{n}^{-}$ to $\theta_{n}^{+}$. Any sequence of points $\left\{\mathbf{x}_{n}\right\} \in\left\{\mathcal{C}_{n}\right\}$ necessarily limits on $\mathbf{x}$. We may take $\mathbf{x}_{n}$ to be a point along $\mathcal{C}_{n}$ where $F\left(\mathbf{x}_{n}\right)=t$.

Finally, suppose that some level set of $F$ is not a topological disc. Let $\mathcal{C}$ be a nontrivial simple closed curve in $F^{-1}(t)$. Span $\mathcal{C}$ by a disc $\mathcal{D}$. We may assume by standard position theory that $\mathcal{D}$ is transverse to $F$. We obtain a contradiction by noting that at an extremum of $F$, interior to $\mathcal{D}$ with value $\neq t$, we have $D F=0$.
We deduce that the ability to solve the BVP gives us $F$ with level sets forming a foliation of $\mathbf{H}^{3}$ by constant mean curvature surfaces.

For the record, one may readily see that this is the Euler-Lagrange equation for the energy functional $\int_{\mathbf{H}^{3}}|D u|+\frac{1}{2} u^{2}$.

\subsection{The Flow}

To exhibit a solution to (3-1), we will consider the parabolic flow given by the equation

$$
F_{t}=\frac{1}{2} \frac{x_{0}|\nabla F|^{2} \Delta F-x_{0} F_{i j} F_{i} F_{j}-2 F_{0}|\nabla F|^{2}}{|\nabla F|^{3}}-F .(3
$$

We seek a steady state solution $F: \mathbf{R}_{\geq 0} \times \mathbf{H}^{3} \rightarrow \mathbf{R}$ to this PDE subject to the conditions that $F$ be nonsingular in space and that it extends continuously to $\mathbf{S}_{\infty}^{2} \backslash \gamma=$ $\Omega_{+} \cup \Omega_{-}$with $\left.F\right|_{\Omega_{+}}=1$ and $\left.F\right|_{\Omega_{-}}=-1$.

For an appropriate choice of initial data, the above scheme seems to converge nicely to a steady state solution. While the initial data may be chosen to have singularities, the flow seems to rectify the singularities. Thus, the putative steady-state solution appears to have no singularities of derivative and thus has level sets foliating $\mathbf{H}^{3}$.

\subsection{Initial Data}

Our initial data for the flow described above will be a smooth function $F(0, X): \mathbf{H}^{3} \rightarrow(-1,1)$ which extends continuously to 1 on $\Omega_{+}$and -1 on $\Omega_{-}$. Since the spaces $\mathbf{H}^{3} \cup \mathbf{S}_{\infty}^{2} \backslash \gamma$ and $\mathbf{D}^{2} \times[-1,1]$, where $\mathbf{D}^{2}$ represents the open unit two-dimensional disc, are diffeomorphic, there always exists such a regular function. The difficulty is in presenting such. Thus, we opt instead for an easily presented choice of $F(0, X)$ which may have singular points. In particular, assuming $\gamma$ has measure 0 , we consider the harmonic extension to $\mathbf{H}^{3}$ of the function which is 1 on $\Omega_{+}$and -1 on $\Omega_{-}$.

The harmonic measure on $\mathbf{H}^{3}$ of a set $\Omega \subset \mathbf{S}_{\infty}^{2}$, as seen from $X=\left(x_{0}, x_{1}, x_{2}\right)$ in an upper half-space model is

$$
\begin{aligned}
& h_{\Omega}(X)= \\
& \frac{1}{\pi} \int_{\Omega}\left(\frac{x_{0}}{x_{0}^{2}+\left(a_{1}-x_{1}\right)^{2}+\left(a_{2}-x_{2}\right)^{2}}\right)^{2} d a_{1} d a_{2} .
\end{aligned}
$$

This is harmonic on $\mathbf{H}^{3}$. If $\Omega$ is not of null measure, then $h_{\Omega}$ is everywhere $>0$, and if $\left(a_{1}, a_{2}\right)$ is in the interior of $\Omega$, then $h_{\Omega}(X) \rightarrow 1$ as $X \rightarrow\left(a_{1}, a_{2}\right)$, while if $\left(a_{1}, a_{2}\right)$ is in the interior of the complement of $\Omega$, then $h_{\Omega}(X) \rightarrow 0$ 
as $X \rightarrow\left(a_{1}, a_{2}\right)$. We will take as our initial data the function

$$
F(0, X)=2 h_{\Omega_{+}}-1
$$

\section{LIMITATIONS}

Not all Jordan curves $\gamma \subset \mathbf{S}_{\infty}^{2}$ can be expected to support a foliation by cmc discs. In fact, Brian White has argued [White 92] that in $\mathbf{R}^{3}$, for a Jordan curve $\gamma$ on a convex body (an extreme curve), the curve is either spanned by a unique, stable, minimal disc or else the collection of all minimal surfaces spanning $\gamma$ is contained in a region (within the convex body) bounded by minimal discs spanning $\gamma$. In the former case, we say that $\gamma$ is stable. White's theorem holds in hyperbolic space as well, and one may pass to Jordan curves at infinity [Velling and White 02].

Now if smooth $\gamma \subset \mathbf{S}_{\infty}^{2}$ is not stable (there exist such), consider the upper and lower minimal discs of the region furnished by White. Call them $\Sigma_{l}$ and $\Sigma_{u}$. They are asymptotic at infinity as they are contained in the convex hull of $\gamma$. In particular, let $\mathbf{x} \in \mathbf{H}^{3}$ be arbitrary, and consider the distance between $\Sigma_{l}$ and $\Sigma_{u}$ in the complement of $B_{r}(\mathbf{x})$. This $\rightarrow 0$ as $r \rightarrow \infty$.

If there is a foliation of $\mathbf{H}^{3}$ by cmc discs spanning $\gamma$, then at least one of $\Sigma_{l}$ and $\Sigma_{u}$ will not be contained in the foliation. Assume without loss of generality that $\Sigma_{u}$ is not a leaf of the foliation. Letting $\Sigma_{0}$ be the minimal disc in the foliation, $\Sigma_{u}$ lies strictly on one side of $\Sigma_{0}$. Again, without loss of generality, assume that $\Sigma_{u}$ lies in the component of $\mathbf{H}^{3} \backslash \Sigma_{0}$ foliated by $\Sigma_{t}(t<0)$. By compactness, there is a least $t$ for which $\Sigma_{u} \cap \Sigma_{t} \neq \emptyset$. For this $t_{0}, \Sigma_{t_{0}}$ and $\Sigma_{u}$ are tangent, with $\Sigma_{u}$ lying between $\Sigma_{0}$ and $\Sigma_{t_{0}}$. This contradicts the maximum principle.

A consequence of the Guan-Spruck result is that all radial graphs in $\mathbf{S}_{\infty}^{2}$ are stable. We therefore restrict our attention to stable curves at infinity.

\section{THE NUMERICAL METHOD}

We have chosen the level set method [Osher and Sethian 88] to compute the flow. The level set method has been successfully used for many calculations related to geometry and curvature flow, for example [Chopp 93, Chopp and Sethian 93, Chopp 94, Angenent et al. 95, Chopp et al. 99]. The level set method is normally associated with the flow of a single interface usually represented by the zero level set; however, in [Chopp 94], the level set method was also used to compute a continuum of interface flows simultaneously.
Each leaf in the foliation will be represented by an isocontour of the function $F$. Translating (3-2) into a level set method formulation, the speed $\Phi$ of the leaf is given by the right-hand side of $(3-2)$ :

$$
\Phi=\frac{1}{2} \frac{x_{0}|\nabla F|^{2} \Delta F-x_{0} F_{i j} F_{i} F_{j}-2 F_{0}|\nabla F|^{2}}{|\nabla F|^{3}}-F .
$$

Inserting (5-1) into the level set method formulation gives an evolution equation for $F$ given by

$$
F_{t}=\Phi|\nabla F| \text {. }
$$

Note that the steady state solutions of (5-2) are the same as for (3-2), while the level set formulation offers a little more stability in the numerical calculations.

The discretization of (5-2) is done using straightforward central difference approximations throughout as in [Chopp and Sethian 93]. The function $F$ is initialized by numerically evaluating $(3-3)$. The grid points located at $\left(0, x_{1}, x_{2}\right)$ are computed to be \pm 1 , and are left fixed throughout the computation. However, this discrete data on the boundary $x_{0}=0$ leads to some grid dependency of the curve $\gamma$. While this can be improved with greater mesh refinement, it cannot be completely removed without resorting to a conforming unstructured mesh and greater computational complexity. Nonetheless, we found that a regular mesh, and allowing for some slight perturbations in $\gamma$, would still produce good approximations for the equilibrium solutions for which we are searching. In order to resolve the large gradients in $F$ for small $x_{0}$ near $\gamma$, we used a more resolved mesh in the $x_{1}$ and $x_{2}$ dimensions than in the $x_{0}$ dimension.

We used this code to study many boundary curves; a few examples of the calculations are presented in Section 6 .

\section{EXAMPLES}

\subsection{The Star-Shaped Case}

In the case where $\Omega_{+}$is a star-shaped plane domain, we present what we consider to be strong evidence that an everywhere regular solution of (3-2) exists as a limit of the flow, starting from our harmonic initial conditions.

First we note the following.

Observation 6.1. When $\Omega_{+}$is star-shaped, the harmonic initial data is an everywhere regular function.

(When $\Omega_{+}$is star-shaped about 0 , the derivative at $\left(x_{0}, x_{1}, x_{2}\right)$ in the upper half-space in direction $\left(x_{0}, x_{1}, x_{2}\right)$ is not zero.) 


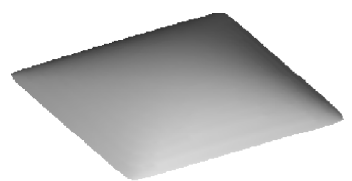

Level $=-0.80$

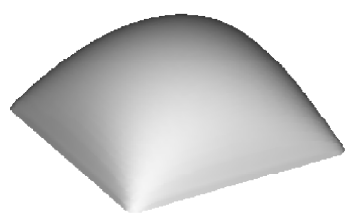

Level $=-0.20$

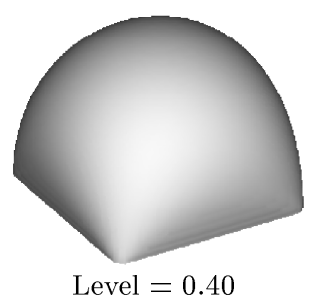

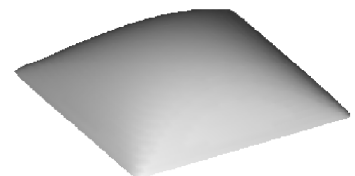

Level $=-0.60$

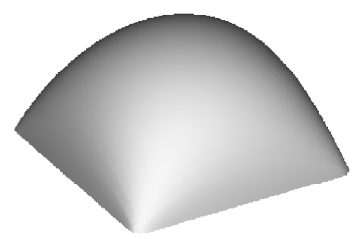

Level $=0.0$

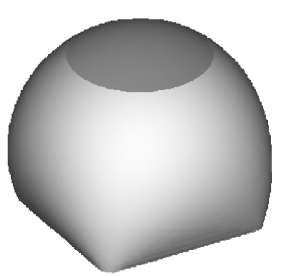

Level $=0.60$

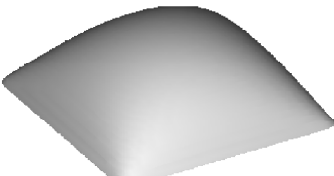

Level $=-0.40$

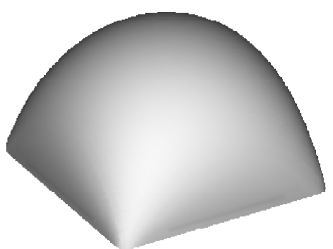

Level $=0.20$

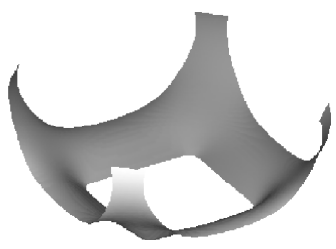

Level $=0.80$

FIGURE 1. Various level sets of equilibrium solution $F$ for $\gamma$ a square.

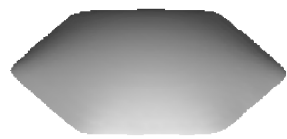

Level $=-0.80$

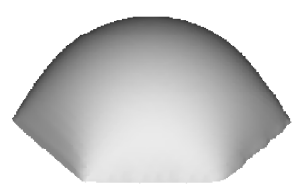

Level $=-0.20$

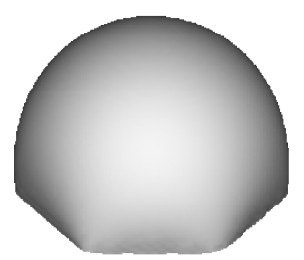

Level $=0.40$

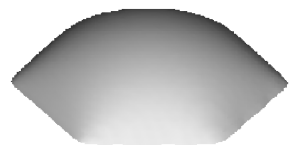

Level $=-0.60$

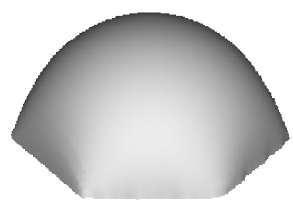

Level $=0.0$

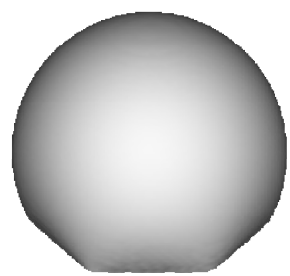

Level $=0.60$

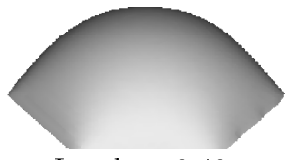

Level $=-0.40$

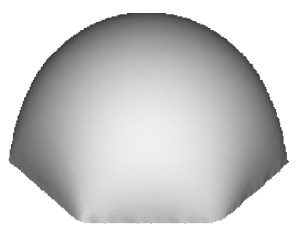

Level $=0.20$

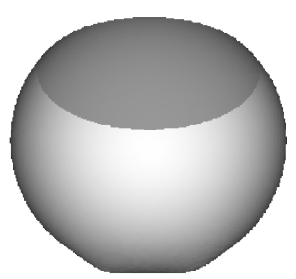

Level $=0.80$

FIGURE 2. Various level sets of equilibrium solution $F$ for $\gamma$ a hexagon. 


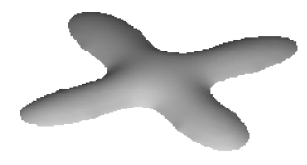

Level $=-0.80$

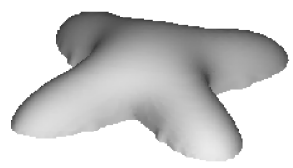

Level $=-0.20$

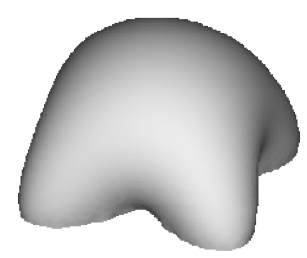

Level $=0.40$

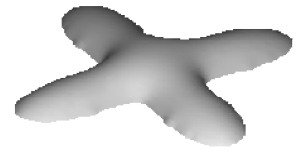

Level $=-0.60$

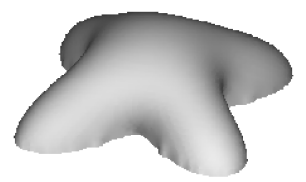

Level $=0.0$

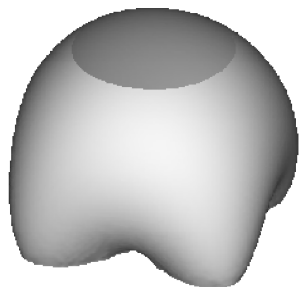

Level $=0.60$

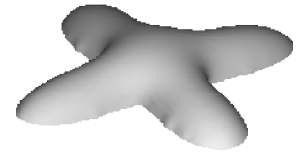

Level $=-0.40$

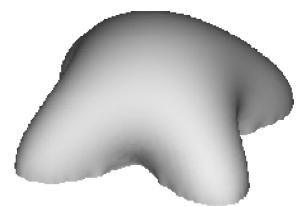

Level $=0.20$

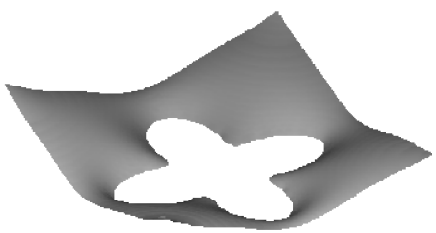

Level $=0.80$

FIGURE 3. Various level sets of equilibrium solution $F$ for $\gamma$ in (6-1).

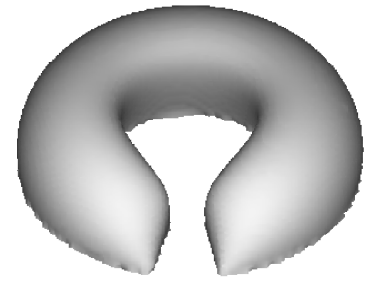

Level $=0.2000$

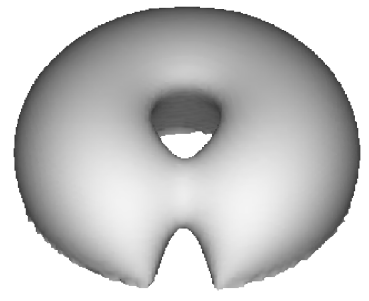

Level $=0.3500$

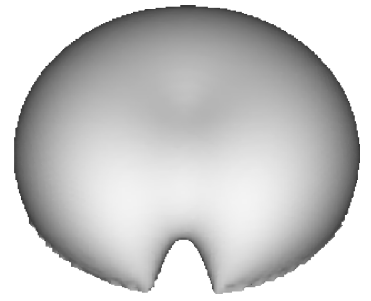

Level $=0.4000$

FIGURE 4. Different level surfaces for the initial data of a notched annulus.

We present here several examples for star-shaped curves $\gamma$. First, we show the case where $\gamma$ is convex-a square and a hexagon. Figure 1 shows the equilibrium solution for when $\gamma$ is a square; Figure 2 shows the solution for the case when $\gamma$ is a hexagon.

Next, a nonconvex, star-shaped curve $\gamma$ was used as an example. Here, $\gamma$ is written in $(r, \theta)$ polar coordinates as

$$
\gamma=\left(1+\frac{1}{2} \cos (4 \theta), \theta\right) .
$$

The equilibrium solution for this curve is shown in Figure 3.

\subsection{The Nonstar-Shaped Case}

Note that the above level sets all consist of a foliation of discs from the initial data until equilibrium. In the following example, we consider the case where the initial data does not consist of a family of discs, rather where, at least initially, not all spanning surfaces have the same topology. In this case, we considered an annulus with a notch taken out.

We note that if the notch is sufficiently narrow, no Möbius transformation of such a region will be starshaped. Hence, we have a fairly simple example where no isometry of $\mathbf{H}^{3}$ will reduce our analysis to the above 
(a)

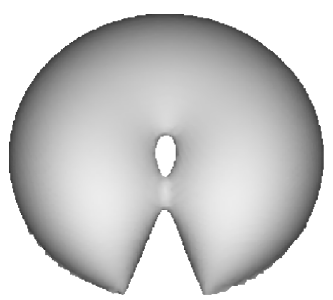

Level $=0.404$

(d)

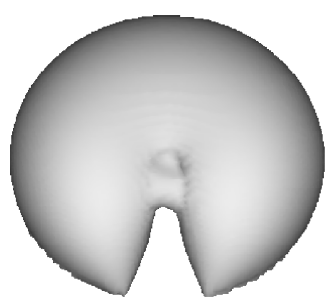

Level $=0.404$ (b)

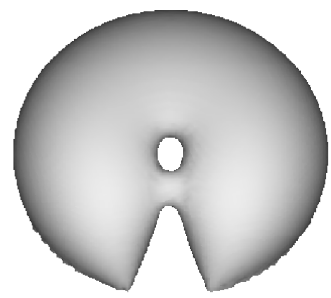

Level $=0.404$

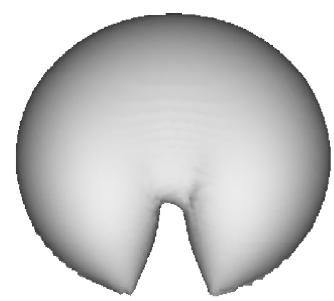

(e)

$$
\text { Level }=0.404
$$

(c)

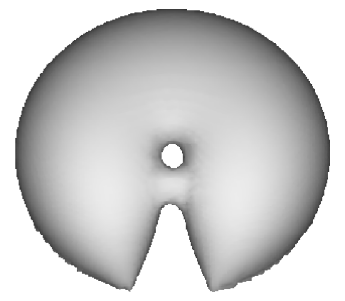

(f)

$$
\text { Level }=0.404
$$

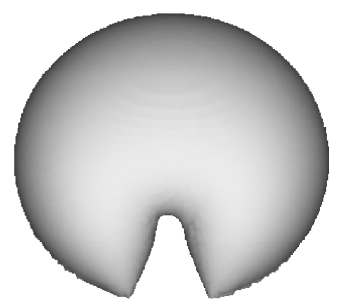

Level $=0.404$

FIGURE 5. Change in topology of prescribed level for a notched annulus. Time steps are (a) $t=0$, (b) $t=0.05$, (c) $t=0.1$, (d) $\mathrm{t}=0.15$, (e) $\mathrm{t}=0.2$, (f) $\mathrm{t}=0.5$.
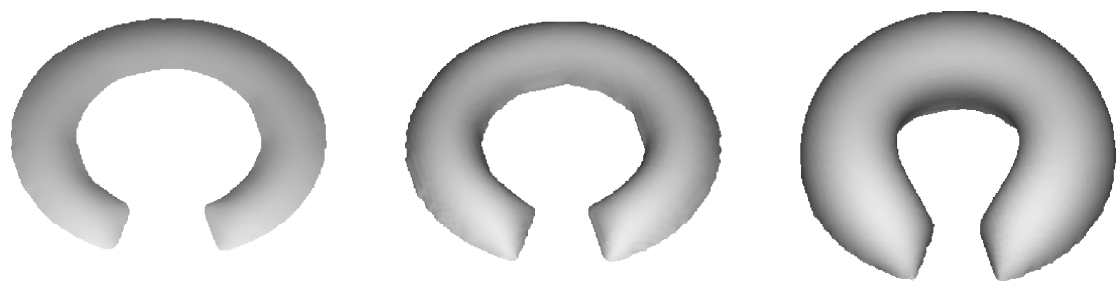

Level $=-0.800$

$$
\text { Level }=-0.300
$$

Level $=0.200$
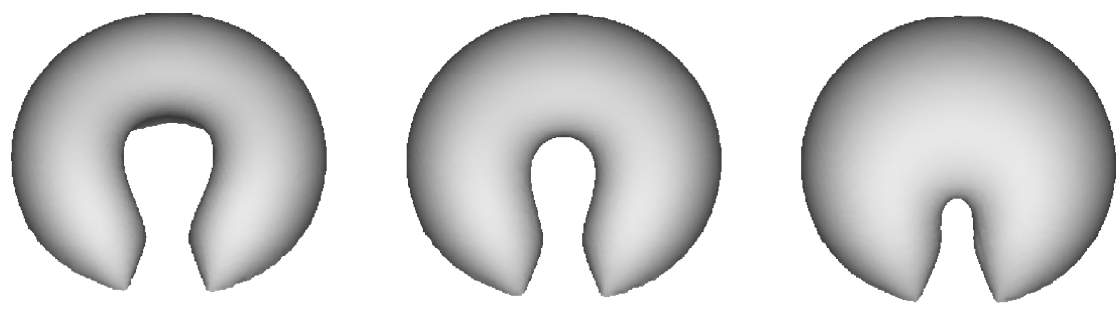

Level $=0.300$

Level $=0.350$

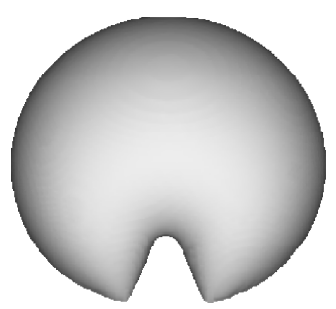

Level $=0.400$

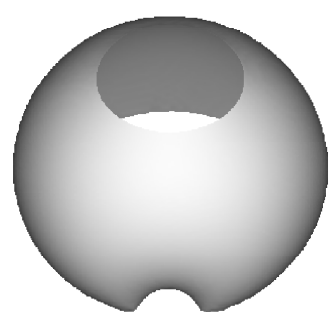

Level $=0.600$

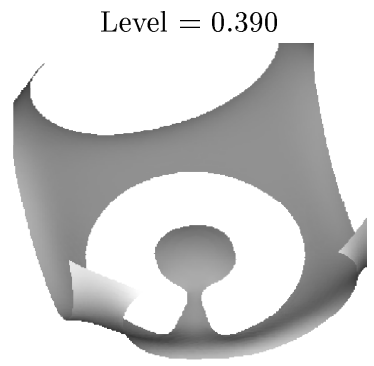

Level $=0.900$

FIGURE 6. Various leafs of the equilibrium $F$ for a notched annulus. 


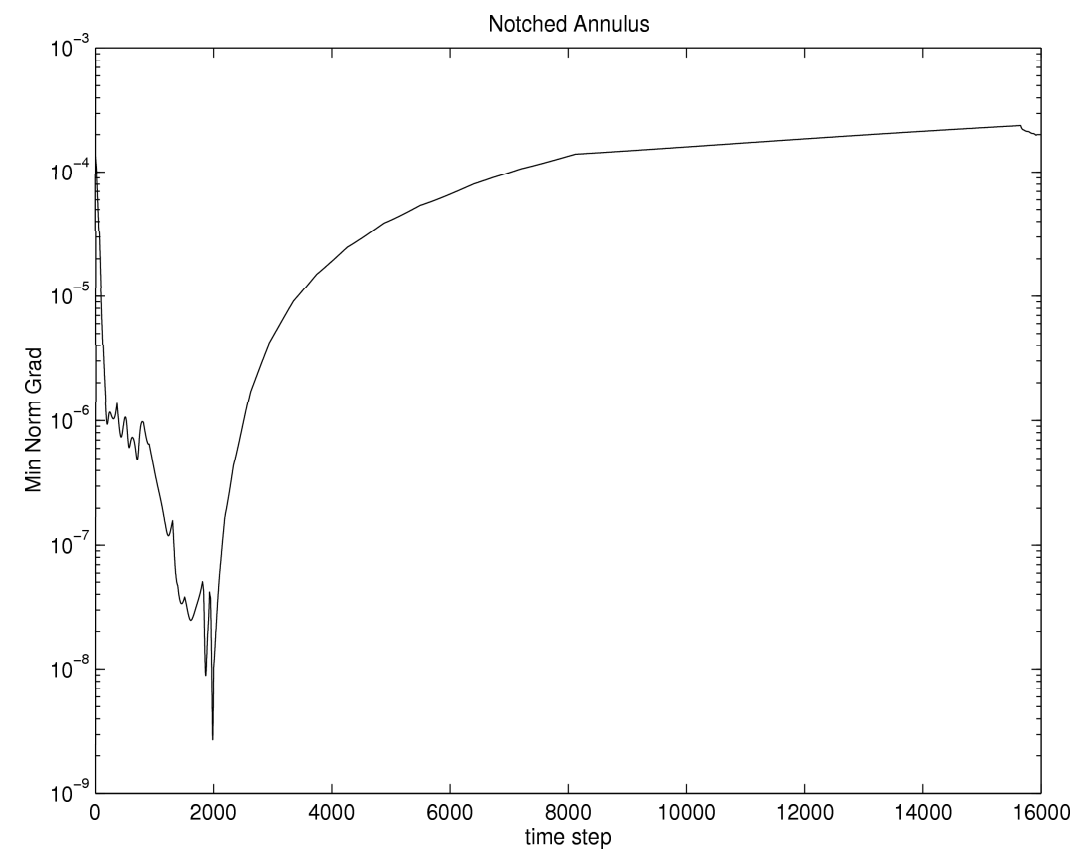

FIGURE 7. Plot of $\min |\nabla F|$ versus time for notched annulus (0.55).

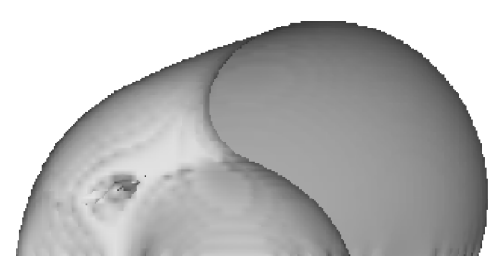

(a) Level $=0.3185$

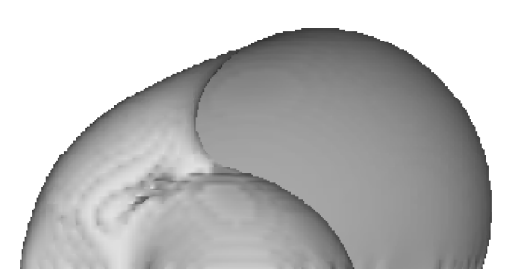

(c) Level $=0.3185$

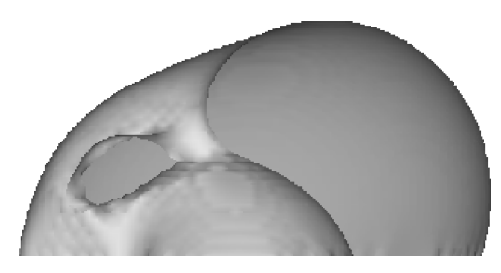

(b) Level $=0.3190$

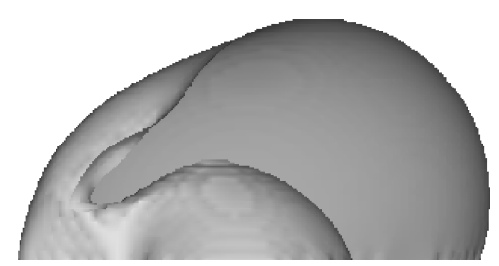

(d) Level $=0.3190$

FIGURE 8. Cutaway view of resolving topology: (a) $t=1.4$, leaf 0.3185 , (b) $t=1.4$, leaf 0.319 , (c) $t=1.5$, leaf 0.3185 , (d) $t=1.5$, leaf 0.319 .

situation. Furthermore, when the notch is sufficiently narrow, our initial data is such that the topology of level sets changes as one progresses from -1 to 1 . Figure 4 illustrates the different topological type level surfaces.

Of particular interest is the resolution of topology. The initial conditions include level sets which are not of disc type, and the evolution resolves this, yielding a steady-state foliation in which all level sets are discs. To illustrate this, we show the evolution of a critical leaf in the foliation for the notched annulus in Figure 5. Initially, this surface has genus 1 , but the hole is filled in the course of the evolution. (We call this "resolution of topology.") The final equilibrium foliation is illustrated in Figure 6.

One important question to be studied from this example is the behavior of $|\nabla F|>0$ during the evolution. On this point, the numerical evidence suggests that $|\nabla F|$ has zeroes while the topology is being resolved, but once 


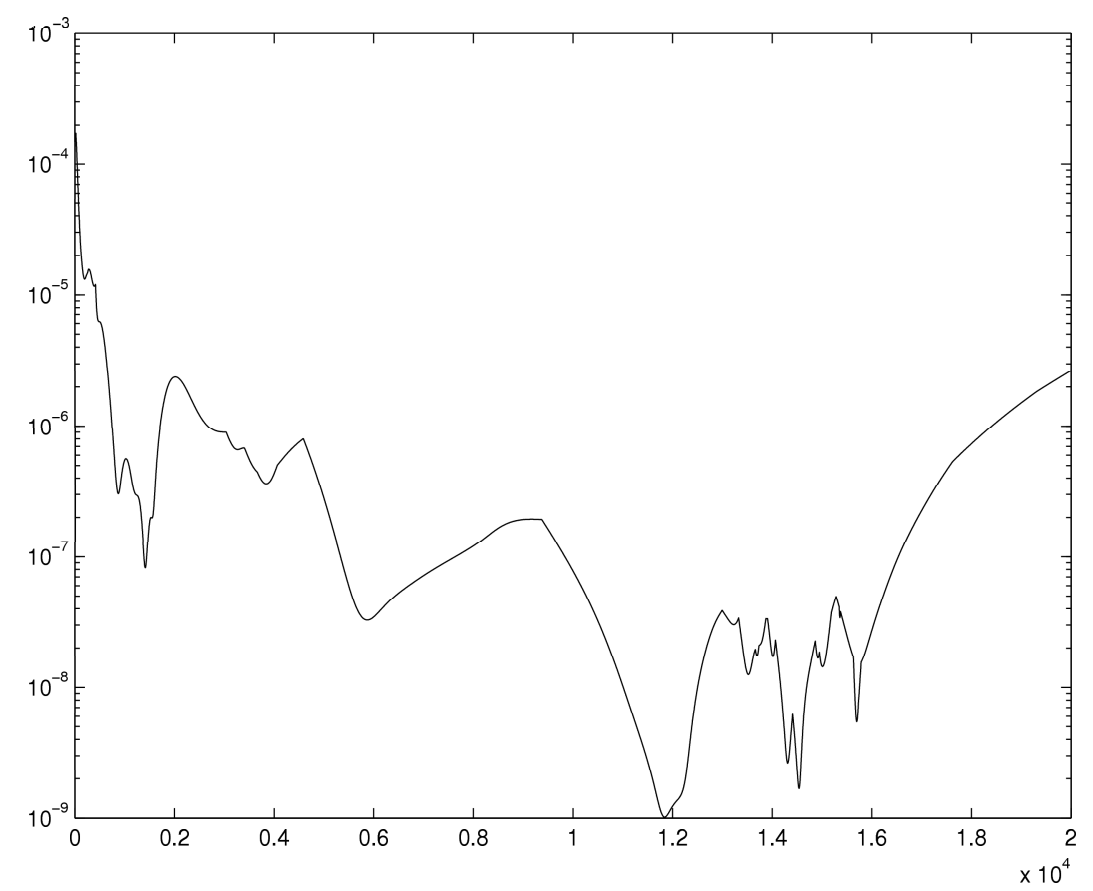

FIGURE 9. Plot of $\min |\nabla F|$ versus time for notched annulus (0.4).

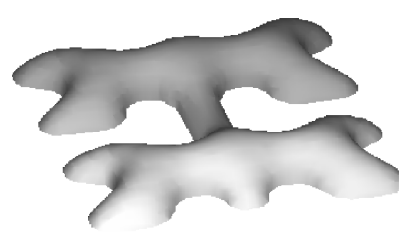

(a) Level $=-0.2000$

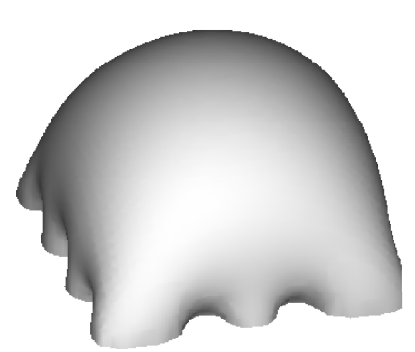

(a) Level $=0.3000$

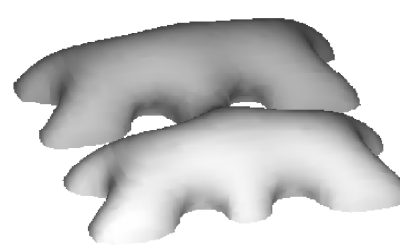

(b) Level $=0.000$

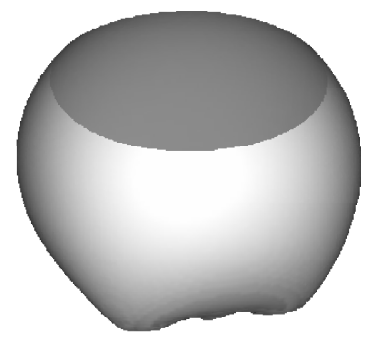

(b) Level $=0.8000$

FIGURE 10. Views of final Peano curve solution: (a) leaf -0.2 , (b) leaf 0 , (c) leaf 0.3 , (d) leaf 0.8.

the topology is resolved, $|\nabla F|$ begins to increase rapidly. A plot of $\min |\nabla F|$ for the notched annulus is shown in Figure 7 where the minimum is taken over the set $\left\{(x, y, z): \sqrt{x^{2}+y^{2}}<R, z>\epsilon\right\}$.

If the notch is made smaller, the time required to resolve the topology gets longer. The notched annulus in
Figures 5-7 had a notch width of 0.55 , and the topology was resolved around $t=0.15$. If the notch is reduced to 0.4 , the topology was not resolved until $t=1.55$, an increase by a factor of ten. Narrowing the notch further renders the calculation computationally too expensive. A cutaway view of the surface as it finally resolves the 


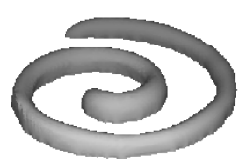

(a) Level $=0.3000$

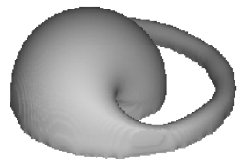

(b) Level $=0.3100$

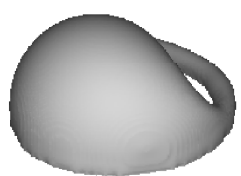

(c) Level $=0.3200$

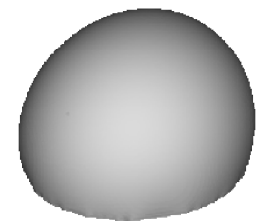

(d) Level $=0.3800$

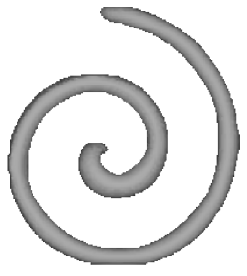

Level $=0.3000$

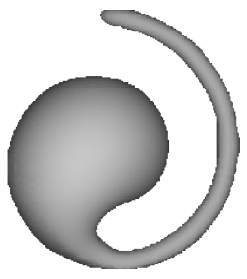

Level $=0.3100$

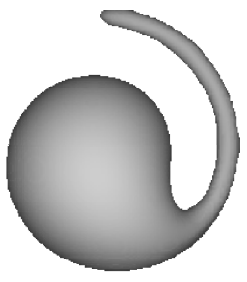

Level $=0.3200$

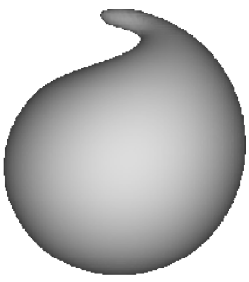

Level $=0.3800$

FIGURE 11. Views of final spiral curve solution: (a) leaf 0.3 , (b) leaf 0.31 , (c) leaf 0.32 , (d) leaf 0.38 .

topology is shown in Figure 8. Again, $\min |\nabla F|$ becomes very small until the topology is resolved, at which point it begins to climb monotonically (Figure 9).

Other nonstar-shaped examples are shown in Figures 10 and 11. In Figure 10, $\gamma$ is a simple Peano curve. In this case, the initial data is a foliation by disks, and no topology change takes place during the evolution.

In Figure 11, $\gamma$ is the boundary of a thin spiral. In this case, the initial data has a similar topological structure to the notched annulus case. The evolution resolves this topology so that the final solution is again a foliation by cmc disks.

\section{CONCLUSION}

We have provided numerical evidence that $\mathbf{H}^{3}$ can be foliated by cmc discs that share the common Jordan curve boundary $\gamma$ in $\mathbf{S}^{2}$. The assumption that the region $\Omega_{+}$ bounded by $\gamma$ be star-shaped, as in [Guan and Spruck $00]$, is likely not necessary.

We illustrated this claim with an example of a notched annulus curve $\gamma$ which cannot be made star-shaped by any Möbius transformation of $\mathbf{S}_{\infty}^{2}$, yet a cmc foliation by discs is still observed. 
The foliation is computed through a level set evolution equation with initial data that closely resembles the desired foliation by $\mathrm{cmc}$ discs. However, the initial data need not be a foliation by discs. During the evolution, some topology changes may occur which transform all leaves into discs as it converges to the final foliation.

The numerical techniques employed for the simulations used the level set method, a well-established numerical method for tracking moving interfaces. It has been widely used for applications in computational differential geometry with success.

While the results presented here do not prove our more general belief that $\mathrm{cmc}$ discs sharing an ideal boundary curve $\gamma$ foliate $\mathbf{H}^{3}$ for stable $\gamma$, the numerical evidence supports it.

\section{REFERENCES}

[Angenent et al. 95] S. Angenent, T. Ilmanen, and D. L. Chopp. "A Computed Example of Nonuniqueness of Mean Curvature Flow in $R^{3}$." Comm. Partial Differential Equations 20:11-12 (1995), 1937-1958.

[Chopp and Sethian 93] D. L. Chopp and J. A. Sethian. "Flow under Curvature: Singularity Formation, Minimal Surfaces, and Geodesics." Journal of Experimental Mathematics 2:4 (1993), 235-255.
[Chopp et al. 99] D. L. Chopp, L. C. Evans, and H. Ishii. "Waiting Time Effects for Gauss Curvature Flows." Indiana U. Math Journal 48:1 (1999), 311-334.

[Chopp 93] D. L. Chopp. "Computing Minimal Surfaces via Level Set Curvature Flow." Journal of Computational Physics 106:1 (1993), 77-91.

[Chopp 94] D. L. Chopp. "Numerical Computation of SelfSimilar Solutions for Mean Curvature Flow." Journal of Experimental Mathematics 3:1 (1994), 1-15.

[Guan and Spruck 00] B. Guan and J. Spruck. "Hypersurfaces of Constant Mean Curvature in Hyperbolic Space with Prescribed Asymptotic Boundary at Infinity." American Journal of Mathematics 122:5 (2000), 1039-1060.

[Nitsche 01] P. Nitsche. "Existence of Prescribed Mean CurvatureGraphs in Hyperbolic Space." Preprint, 2001.

[Osher and Sethian 88] S. Osher and J. A. Sethian. "Fronts Propagating with Curvature-Dependent Speed: Algorithms Based on Hamilton-Jacobi Formulations." Journal of Computational Physics 79:1 (1988), 12-49.

[Velling and White 02] J. A. Velling and B. White. Personal communication, 2002.

[Velling 99] J. A. Velling. "Existence and Uniqueness of Complete Constant Mean Curvature Surfaces at Infinity of $H^{3}$." Journal of Geometric Analysis 9:3 (1999), 457489.

[White 92] B. White. "On the Topological Type of Minimal Submanifolds." Topology 31:2 (1992), 445-448.

David Chopp, Department of Engineering Sciences and Applied Mathematics, Northwestern University, Evanston, IL 60208 (chopp@northwestern.edu)

John A. Velling, Department of Mathematics, Brooklyn College, Brooklyn, NY 11210 (jvelling@brooklyn.cuny.edu)

Received October 4, 2002; accepted in revised form March 25, 2003. 